\title{
Three-Dimensional Simulations of the Anisotropic Etching Profile Evolution for Producing Nanoscale Devices
}

\author{
B. Radjenović And M. Radmilović-Radjenović \\ Institute of Physics, Pregrevica 118, 11080 Belgrade, Serbia \\ (Received February 27, 2010; in final form November 18, 2010)
}

\begin{abstract}
Refined control of etched profiles is one of the most important tasks of micro (nano) electro mechanical systems manufacturing process. In spite of its wide use, the simulation of etching for micro (nano) electro mechanical systems applications has been so far a partial success only, although a great number of commercial and academic research tools dedicated to this problem are developed. In this paper we describe an application of the sparse field method for solving level set equations in $3 \mathrm{D}$ anisotropic wet etching of silicon with potassium hydroxide $(\mathrm{KOH})$. Angular dependence of the silicon etching rate is determined on the basis of the silicon crystal symmetry properties. Some examples illustrating developed methodology are given.
\end{abstract}

PACS: 81.65.Cf

\section{Introduction}

The inherently multi-physical and multi-disciplinary design of micro (nano) electro mechanical systems $\mathrm{M}(\mathrm{N})$ EMS devices requires new design methodologies including the integration of modelling, design, and simulation for M(N)EMS as early as possible in the course of the different life-cycle phases [1].

Level set method, introduced by Osher and Sethian [2], is a powerful technique for analyzing and computing moving fronts in a variety of different settings. The level sets are used in image processing, computer vision, computational fluid dynamics, material science, and many other fields. Detailed exposition of the theoretical and numerical aspects of the method, and applications to different areas can be found in books [2] and [3]. During last several years several variants of level set methods have been developed with application to microelectronic devices fabrication problems. The profile surface evolution in etching, deposition and lithography development is a significant challenge for implementation of numerical methods in front tracking. The level set methods for evolving interfaces are specially designed for profiles which can develop sharp corners, change of topology and undergo orders of magnitude changes in speed. They are based on the Hamilton-Jacobi type equation for the level set function using techniques developed for solving hyperbolic partial differential equations. In this paper we describe shortly the level set method as well as sparse field method for solving the level set equations.

The sparse-field method itself, developed by Whitaker [4], and broadly used in image processing community, is an alternative to the usual combination of narrow band and fast marching procedures for the computationally effective solving of the level set equations. After that we analyze the case of non-convex Hamiltonians and its application in the simulations of the etching profile evolution during anisotropic wet etching of silicon with $\mathrm{KOH}$ etchant in more details.

\section{Level set method}

The basic idea behind the level set method is to represent the surface in question at a certain time $t$ as the zero level set (with respect to the space variables) of a certain function $\varphi(t, \boldsymbol{x})$, the so-called level set function. The initial surface is given by $\{\boldsymbol{x} \mid \varphi(0, \boldsymbol{x})=0\}$. The evolution of the surface in time is caused by "forces" or fluxes of particles reaching the surface in the case of the etching process. The velocity of the point on the surface normal to the surface will be denoted by $R(t, \boldsymbol{x})$, and is called velocity function. For the points on the surface this function is determined by physical models of the ongoing processes; in the case of etching by the fluxes of incident particles and subsequent surface reactions. The velocity function generally depends on the time and space variables and we assume that it is defined on the whole simulation domain. At a later time $t>0$, the surface is as well the zero level set of the function $\varphi(t, \boldsymbol{x})$, namely it can be defined as a set of points $\left\{\boldsymbol{x} \in \Re^{n} \mid \varphi(t, \boldsymbol{x})=0\right\}$. This leads to the level set equation

$$
\frac{\partial \varphi}{\partial t}+R(t, \boldsymbol{x})|\nabla \varphi|=0,
$$

in the unknown function $\varphi(t, \boldsymbol{x})$, where $\varphi(0, \boldsymbol{x})=0$ determines the initial surface. Having solved this equation the zero level set of the solution is the sought surface at all later times. Actually, this equation relates the time 
change to the gradient via the velocity function. In the numerical implementation the level set function is represented by its values on grid nodes, and the current surface must be extracted from this grid. In order to apply the level set method a suitable initial function $\varphi(0, \boldsymbol{x})$ has to be defined first. The natural choice for the initialization is the signed distance function of a point from the given surface.

Here we will present a simple example illustrating etching profile evolution. The initial profile surface is a rectangle dip. In Fig. 1 the results obtained by the calculation performed with constant velocity function $R=R_{0}$ (purely isotropic etching case) are shown. It is supposed that only the bottom surface could be etched; i.e. that the top and the vertical surfaces belong to photoresist layer. Behavior of the etching profile is as expected. Equation (1) can be rewritten in the Hamilton-Jacobi form [5]:

$$
\frac{\partial \varphi}{\partial t}+H(\nabla \varphi(t, x))=0
$$

where Hamiltonian is given by $H=V(t, \boldsymbol{x})|\nabla \varphi(t, \boldsymbol{x})|$ (in this context the term "Hamiltonian" denotes a Hamiltonian function, not an operator). If the surface velocity $R(t, \boldsymbol{x})$ does not depend on the level set function $\varphi(t, \boldsymbol{x})$ itself, this condition is usually satisfied. In that case, we can say that the problem is of free boundary type. In that case the spatial derivatives of $\varphi$ can be approximated using the Engquist-Osher upwind finite difference scheme, or by higher-order essentially non-oscillatory (ENO) and weighted essentially non-oscillatory (WENO) discretization schemes, that requires the values of this function at the all grid points considered. The resulting semidiscrete equations can be solved using explicit Euler method, or more precisely by total-variation diminishing (TVD) Runge-Kutta time integration procedure (see Refs. [2] and [3] for the details).
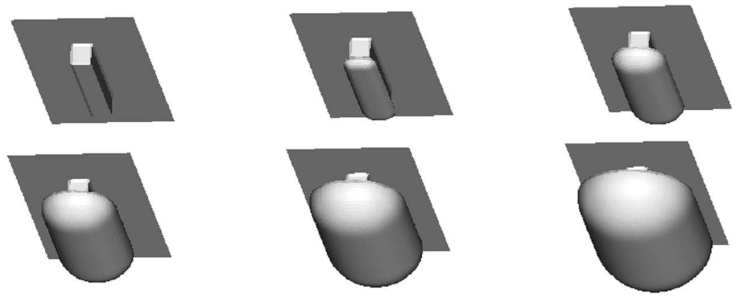

Fig. 1. Isotropic etching - feature profiles evolution for $R=R_{0}$ at equidistant (reduced) etching time moments.

The non-convex Hamiltonians are characteristic for anisotropic wet etching, plasma etching and deposition simulations. The upwind (ENO, WENO) finite difference scheme cannot be used in the case of non-convex Hamiltonians. The simplest scheme that can be applied in these cases is the Lax-Friedrichs, one which relies on the central difference approximation to the numerical flux function, and preserves monotonicity through a second- -order linear smoothing term

$$
\begin{aligned}
\varphi_{i j k}^{n+1} & =\varphi_{i j k}^{n}-\Delta t \\
\times & {\left[H\left(\frac{D_{i j k}^{-x}+D_{i j k}^{+x}}{2}, \frac{D_{i j k}^{-y}+D_{i j k}^{+y}}{2}, \frac{D_{i j k}^{-z}+D_{i j k}^{+z}}{2}\right)\right.} \\
- & \frac{1}{2} \alpha_{x}\left(D_{i j k}^{+x}-D_{i j k}^{-x}\right)-\frac{1}{2} \alpha_{y}\left(D_{i j k}^{+y}-D_{i j k}^{-y}\right) \\
- & \left.\frac{1}{2} \alpha_{z}\left(D_{i j k}^{+z}-D_{i j k}^{-z}\right)\right],
\end{aligned}
$$

where $D_{i j k}^{+x(y, z)}$ and $D_{i j k}^{-x(y, z)}$ are usual forward and backward differences

$$
\begin{array}{ll}
D_{i j k}^{+x}=\frac{\varphi_{i+1, j, k}^{n}-\varphi_{i, j, k}^{n}}{\Delta x}, & D_{i j k}^{-x}=\frac{\varphi_{i, j, k}^{n}-\varphi_{i-1, j, k}^{n}}{\Delta x}, \\
D_{i j k}^{+y}=\frac{\varphi_{i, j+1, k}^{n}-\varphi_{i, j, k}^{n}}{\Delta y}, & D_{i j k}^{-y}=\frac{\varphi_{i, j, k}^{n}-\varphi_{i, j-1, k}^{n}}{\Delta y}, \\
D_{i j k}^{+z}=\frac{\varphi_{i, j, k}^{n}-\varphi_{i, j, k+1}^{n}}{\Delta z}, & D_{i j k}^{-z}=\frac{\varphi_{i, j, k}^{n}-\varphi_{i, j, k-1}^{n}}{\Delta z},
\end{array}
$$

and $\alpha_{x}\left(\alpha_{y}, \alpha_{z}\right)$ is a bound on the partial derivative of the Hamiltonian with respect to the first (second, third) argument

$$
\begin{aligned}
& \alpha_{x}=\max \left|\frac{\partial H}{\partial \varphi_{x}}\right|, \quad \alpha_{y}=\max \left|\frac{\partial H}{\partial \varphi_{y}}\right|, \\
& \alpha_{z}=\max \left|\frac{\partial H}{\partial \varphi_{z}}\right| .
\end{aligned}
$$

It was shown that it is possible to use the Lax-Friedrichs scheme in conjunction with the sparse field method, and to preserve sharp interfaces and corners by optimizing the amount of smoothing in it [6].

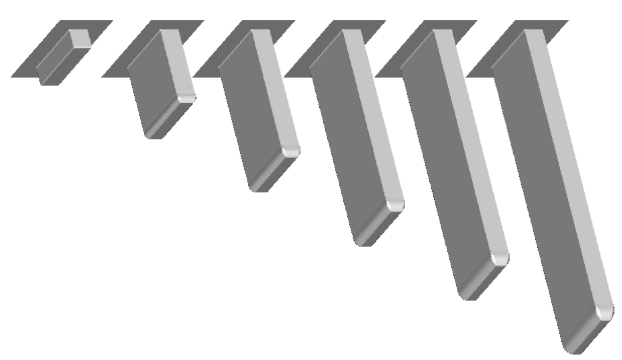

Fig. 2. Anisotropic etching - feature profiles evolution for $R=R_{0} \cos \theta$ at equidistant (reduced) etching time moments.

In Fig. 2 the evolution of the etching profile, when etching rate is proportional to trigonometrical function $\cos \theta$, where $\theta$ is the angle between surface normal and vertical direction (z-axes), is presented. This is the simplest form of angular dependence that leads to a non-convex 
Hamiltonian, but it describes the ion enhanced chemical plasma etching process correctly [7]. In this case we expect that the horizontal surfaces move downward, while the vertical ones stay still. This figure shows that it, with optimal amount of smoothing, gives minimal rounding of sharp corners, while preserving the numerical stability of the calculations. Actually, this is one of the most delicate problems in the etching profile simulations.

\section{Anisotropic wet etching of silicon}

Although silicon etching techniques are currently undergoing a revolution driven by the incorporation of plasma etching process, anisotropic wet chemical etching is still the most widely used processing technique in silicon technology [1]. It is also referred to as "bulk micromachining", since in this technology the body of the silicon wafer is etched away. The wide presence of anisotropic etching is not only due to its ease of use and low cost, but also to the fact that it provides rather smooth surfaces with no physical damage to the bulk structure of the material. Very complicated three-dimensional structures can be formed by this technique; it enables controlled undercutting of suspended structures, not possible by other microfabrication techniques. Etch rates significantly depend upon many process variables: the etchant concentration, the temperature, the presence of metal impurities in solution, the use of alcohols and oxidizing agents as additives in the etch bath, the concentration of silicon in solution, the presence of dissolved oxygen in solution, the use of biasing potentials and stirring, and the level of oxygen impurities in the bulk of the material, etc. The anisotropy of the etching process refers to the orientation dependence of the etch rate, that is the ratio of the distance advanced by the surface to the etching time.

As pointed out earlier, in order to simulate the time evolution of three-dimensional etching profiles it is essential that exact etch rates in all directions are known. The etching rates for only a few principal axes are known, but they can be used to determine rate value in an arbitrary direction by an interpolation procedure. The problem of etching rate interpolation is equivalent to function interpolation over a sphere in 3D. For accuracy, the etching rate model must interpolate through the given etching rates and directions while maintaining $C^{0}$ continuity, since $C^{1}$ is too high, as empirical studies have shown cusps in etching rate diagrams. Here we shall use etching rate model developed by Hubbard [8], that satisfies these conditions. Of course, this is not the only possibility; the problem of finding the optimal interpolation method is out of scope of this paper. It is supposed that $x, y$ and $z$ axes are aligned with [100], [010] and [001] crystal directions, respectively.

\section{Simulation results}

Potassium hydroxide $(\mathrm{KOH})$ is the most common and the most important chemical etchant, because of its excellent repeatability and uniformity in fabrication, and its low production cost. In actual calculations we made use of measured [9] etching rates in [100], [110], [111] and [311] crystal directions, for $30 \% \mathrm{KOH}$ concentration at $70^{\circ} \mathrm{C}\left(R_{111}=0.005 \mu \mathrm{m} / \mathrm{min}, R_{100}=0.797 \mu \mathrm{m} / \mathrm{min}\right.$, $R_{110}=1.455 \mu \mathrm{m} / \mathrm{min}$ and $\left.R_{311}=1.436 \mu \mathrm{m} / \mathrm{min}\right)$. The actual shapes of the initial surfaces are described using simple geometrical abstractions. In the beginning of the calculations these descriptions are transformed into the initial level set functions using the fast marching method [2]. Our implementation is based on ITK library [10]. The classes describing the level set function and the level set filter are reimplemented according to the procedures for treating non-convex Hamiltonians described in the previous section.
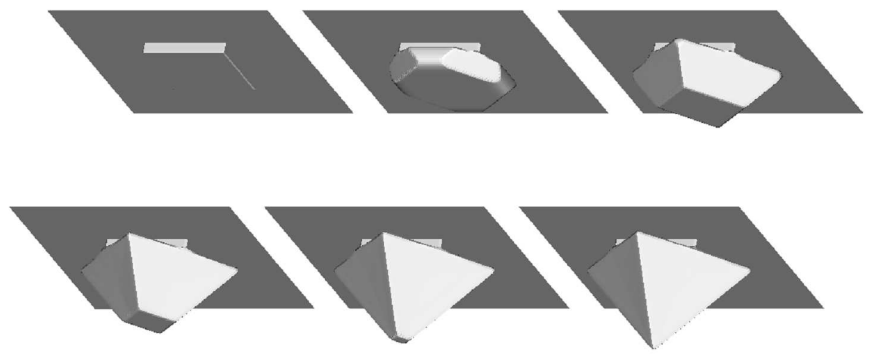

Fig. 3. Etching through a square mask in $\{100\}$ plane aligned to $\langle 100\rangle$ directions. Profiles at six equidistant (reduced) time moments.
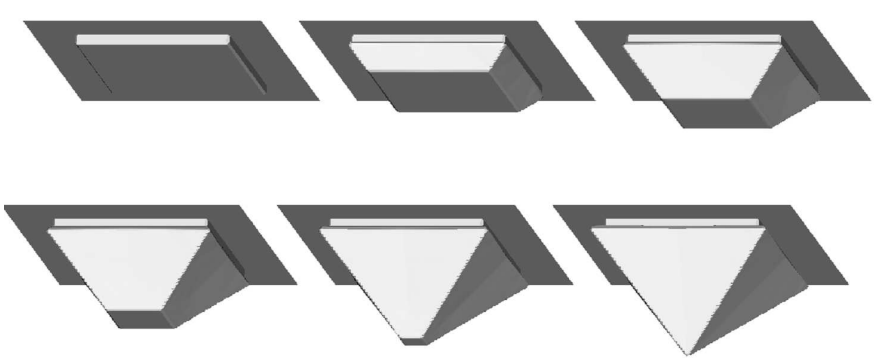

Fig. 4. Etching through a square mask in $\{100\}$ plane aligned to $\langle 110\rangle$ directions. Profiles at six equidistant (reduced) time moments.

The first example is etching through square openings in the $\{100\}$ silicon plane with edges aligned to $\langle 100\rangle$ and $\langle 110\rangle$ directions. The time evolution of the etched profiles is shown in Figs. 3 and 4, respectively. Formation of the V-shaped cavities consisting of only $\{111\}$ planes is reproduced correctly.

\section{Conclusions}

In this paper there is shown that profile evolution during anisotropic wet etching of silicon can be described by the non-convex Hamiltonian arising in the HamiltonJacobi equation for the level set function. Etching rate angular dependence is calculated on the base of the silicon symmetry properties, by means of the interpolation technique using experimentally obtained values of the 
principal [100], [110], [111], and high index [311] directions in $\mathrm{KOH}$ solutions $\mathrm{KOH}$ solutions $[11,12]$. The calculations are performed using an extension of the sparse field method for solving three-dimensional (3D) level set equations in the case of non-convex Hamiltonians. The obtained results show that level set method can be used as an effective tool for wet etching process modelling, and that is a viable alternative to the cellular automata method which now prevails in the simulations of the wet etching process [13].

\section{References}

[1] M. Elwenspoek, H.V. Jansen, Silicon Micromachining, Cambridge University Press, Cambridge, UK 1998.

[2] J. Sethian, Level Set Methods and Fastmarching Methods: Evolving Interfaces in Computational Geometry, Fluid Mechanics, Computer Vision and Materials Sciences, Cambridge University Press, Cambridge, UK 1998.

[3] S. Osher, R. Fedkiw, Level Set Method and Dynamic Implicit Surfaces, Springer-Verlag, New York, NY 2003.
[4] R. Whitaker, Int. J. Comp. Vision 29, 203 (1998).

[5] L. Evans, Partial Differential Eqautions, American Mathematical Society, Providence, RI 1998.

[6] B. Radjenović, J.K. Lee, M. Radmilović-Radjenović, Computer Phys. Commun. 174, 127 (2006).

[7] J.A. Sethian, D. Adalsteinsson, IEEE Trans. Semicond. Dev. 10, 167 (1996).

[8] T.J. Hubbard, Ph.D. Thesis, California Institute of Technology, 1994.

[9] K. Sato, M. Shikida, Y. Matsushima, T. Yamashiro, Sensors Actuators A 64, 87 (1988).

[10] NLM Insight Segmentation and Registration Toolkit, http://www.itk.org .

[11] M. Buerger, Elementary Crystallography, Wiley, New York, Chapman \& Hall, London 1956.

[12] M. Shikida, K. Sato, K. Tokoro, D. Uchikawa, Sensors Actuators A 80, 179 (2000).

[13] B. Radjenović, M. Radmilović-Radjenović, M. Mitrić, Appl. Phys. Lett. 89, 213102 (2006). 